\title{
Rendimiento, calidad y biogás in vitro en pasto Megathyrsus maximus cv. Aruana con diferente fertilización
}

\section{Performance, quality an in vitro biogas in grass Megathyrsus maximus cv. Aruana with different fertilization}

\begin{abstract}
Paulino Sánchez-Santillán 1(D) Claudia Yanet Wilson-García $*$ (D) Nelson Erik López-Zerón 3 [D Luis Antonio Saavedra-Jiménez 1 (D) María de los Ángeles MaldonadoPeralta 1 (D)

Mara Yuciry Melo-Trani1 1

${ }^{1}$ Universidad Autónoma de Guerrero, Facultad de Medicina Veterinaria y Zootecnia No. 2. Carretera federal Acapulco-Pinoteca Nacional Km 197 CP. 41940. Cuajinicuilapa, Guerrero, México.

${ }^{2}$ Universidad Autónoma Chapingo, Sede San Luis Acatlán, San Luis Acatlán CP. 41600. Guerrero, México. ${ }^{3}$ Centro de Bachillerato Tecnológico Agropecuario No. 178. San Luis Acatlan-Horcasitas $\mathrm{Km} \quad 5$, Playa Larga, CP. 41603. San Luis Acatlán, Guerrero, México.
\end{abstract}

*Autor de correspondencia:

cwilsong@chapingo.mx.

Artículo científico

Recibido: 08 de marzo 2021

Aceptado: 25 de septiembre 2021

Como citar: Sánchez-Santillán P, Wilson-García CY, LópezZerón NE, Saavedra-Jiménez LA Maldonado-Peralta MA, Melo-Trani MY (2021) Rendimiento, calidad y biogás in vitro en pasto Megathyrsus maximus cv. Aruana con diferente fertilización. Ecosistemas y Recursos Agropecuarios Núm. Esp. II: e2957. DOI: 10.19136/era.a8nII.2957
RESUMEN. El objetivo fue evaluar el rendimiento, la composición bromatológica y producción de biogás in vitro con fertilización orgánica e inorgánica, para determinar la edad de corte óptimo del pasto Megathyrsus maximus (Jacq.) B.K. Simon \& S.W.L. Jacobs Cv. aruana. Los tratamientos fueron: fertilización con composta, lombricomposta, composta más lixiviado y fertilización química. El diseño experimental fue bloques completos al azar, en arreglo de medidas repetidas en el tiempo con tres repeticiones, se realizaron muestreos a los 20,35 , $50,65,80$ y 95 días después del corte (DDC) de uniformización. Las variables evaluadas fueron acumulación de materia seca, composición morfológica, altura, fibra detergente neutro, fibra detergente acido, materia orgánica, proteína cruda, cenizas y producción de biogás in vitro. Los mayores rendimientos fueron a los 80 DDC con los tratamientos químico y composta más lixiviado con 24013 y 10 $974 \mathrm{~kg} \mathrm{MS} \mathrm{ha}^{-1}$, respectivamente. En los tratamientos orgánicos se encontró mayor relación hoja: tallo comparado con el químico en todas las fechas evaluadas. Los tratamientos mostraron descenso del porcentaje de proteína cruda conforme avanzó la edad de rebrote. A los 50 y 95 DDC, el pasto aruana fertilizado con lombricomposta o composta más lixiviado produjeron 2.8 y 2.0 veces mayor biogás que el fertilizado con químico o composta. La fecha óptima de corte para el pasto aruana para el tratamiento químico y composta más lixiviado es a los 50 y 65 DDC, mientras que para lombricomposta y composta es a los 65 DDC.

Palabras clave: Bromatológico, composta, lixiviado, lombricomposta, Panicum maximum.

ABSTRACT. The objective was to evaluate the yield, bromatological composition and biogas production in vitro with organic and inorganic fertilization, to determine the optimal cutting age of Megathyrsus maximus (Jacq.) B.K. Simon \& S.W.L. Jacobs Cv. aruana. The treatments were; fertilization with compost, vermicompost, compost plus leachate and chemical fertilization. The experimental design was complete randomized blocks, in an arrangement of measures repeated in time with three repetitions, samplings were carried out at 20,35, 50, 65, 80 and 95 days after the cut-off (DDC) of standardization. The variables evaluated were accumulation of dry matter, morphological composition, height, neutral detergent fiber, acid detergent fiber, organic matter, crude protein, ash, and in vitro biogas production. The highest yields were at $80 \mathrm{DDC}$ with the chemical treatments and the most leached compost with 24013 and $10974 \mathrm{~kg} \mathrm{DM} \mathrm{ha}^{-1}$, respectively. In the organic treatments, a higher leaf: stem ratio was found compared to the chemical one in all the evaluated dates. The treatments showed a decrease in the percentage of crude protein as the regrowth age advanced. At 50 and 95 DDC, the aruana grass fertilized with vermicompost or more leached compost produced 2.8 and 2.0 times more biogas than the one fertilized with chemical or compost. The optimal cutting date for aruana grass for the most leached chemical treatment and compost is 50 and $65 \mathrm{DDC}$, while for vermicompost and compost it is $65 \mathrm{DDC}$.

Key words: Bromatological, compost, leached, vermicompost, Panicum maximum. 


\section{INTRODUCCIÓN}

La mayoría de los sistemas de producción de rumiantes en las regiones tropicales y subtropicales de México, se desarrollan bajo condiciones de pastoreo extensivo, con gramíneas nativas, con producción de forraje irregular, tanto en cantidad como en calidad (Améndola et al. 2005). Por lo anterior, se han introducido gramíneas mejoradas para elevar la productividad de las praderas y producción animal (Enríquez-Quiroz et al. 2011). Al respecto, Ramírez-Meléndez at al. (2020) y Quero (2013) mencionan que es necesario promover la evaluación y comprobación de los atributos de los mejores genotipos; ya que se han reportado elevados porcentajes de variación genética en la producción dentro de cada especie, por lo que es recomendable evaluar los diferentes cultivares. Megathyrsus maximus es una especie africana, ampliamente cultivada como forrajera en las regiones tropicales y subtropicales en América del Sur, al oeste de la India, y al sur y este de Asia. El cultivar aruana se caracteriza por requerir suelos fértiles para su óptimo desarrollo, el forraje es usado principalmente en el ganado ovino y caprino, debido a su bajo porte, no obstante, también con los bovinos y los equinos se observan excelentes resultados. Su producción es de 15 a $20 \mathrm{t}$ $\mathrm{MS}^{-1}$ ha $^{-1}$ año $^{-1}$ (Castrejón et al. 2017). Los factores determinantes de la producción y composición química de los forrajes se pueden dividir en dos grupos; factores propios de la planta como son especie, edad y composición morfológica, y los de tipo ambiental como temperatura, radiación solar, precipitación, fertilidad y tipo de suelo (EnríquezQuiroz et al. 2011). Así mismo, debido a que la gran mayoría de los ganaderos no fertiliza, las praderas sufren de deficiencias de $\mathrm{N}$ y otros minerales (QueroCarrillo et al. 2010). Sin embargo, un alto número de evaluaciones en gramíneas tropicales se han efectuado con fertilización inorgánica, pero son casi nulas las evaluaciones con fertilizantes orgánicos (Jiménez et al. 2010). Los fertilizantes inorgánicos aumentan el nitrógeno disponible para las plantas, pero poco se han estudiado los abonos orgánicos en praderas, además los inorgánicos no producen cam- bios importantes en la textura y población bacteriana del suelo a diferencia de los de tipo orgánico (Flores et al. 2012). Esto afecta la calidad de los forrajes, la cual se relaciona directamente con el crecimiento, la producción de leche y carne de los rumiantes (Muñoz et al. 2016). En el trópico las condiciones ambientales provocan un rápido crecimiento de los pastos, los cuales muestran alta concentración de pared celular, baja digestibilidad y disponibilidad de energía, impidiendo el uso óptimo de los nutrientes por los animales; por lo que es necesario realizar el análisis bromatológico y pruebas de producción de gas in vitro, in situ e in vivo para utilizar los forrajes en el momento óptimo de cosecha (Sánchez et al. 2005) e identificar sí el manejo agronómico realizado como la fertilización incide en estas variables. AlmarazBuendía et al. (2019) realizaron un estudio con los pastos bermuda, mulato, insurgente, estrella, bramilla, llanero, mombaza, pará y pangola a 56 días de rebrote sin fertilización, encontrando que el pasto mombaza obtuvo las mejores características bromatológicas, las mejores producciones de gas total, donde el metano representó $40.3 \%$ y las mayores degradaciones de las fibras detergentes neutro y ácido. En el caso de pasto aruana no hay información sobre producción de biogás evaluado en diferentes tipos de fertilización, por anterior el objetivo del presente trabajo fue evaluar el rendimiento, la composición bromatológica y producción de biogas in vitro con fertilización orgánica e inorgánica, para determinar la edad de corte óptimo del pasto $M$. maximus cv. Aruana.

\section{MATERIALES Y MÉTODOS}

El experimento se estableció en la Sede San Luis Acatlán de la Universidad Autónoma Chapingo, ubicada en el municipio de San Luis Acatlán en la Región Costa Chica, Guerrero, coordenadas $16^{\circ} 51$ $28.99 \mathrm{LN}$ y $98^{\circ} 4326.13 \mathrm{LO}$, a $311 \mathrm{msnm}$. El sitio presenta una temperatura promedio anual de 27.9 ${ }^{\circ} \mathrm{C}$, siendo el mes de mayo el más caluroso con 29.7 ${ }^{\circ} \mathrm{C}$ y enero el de menor temperatura con $26.7^{\circ} \mathrm{C}$. La precipitación media anual es de $1395 \mathrm{~mm}$, con mayor 
precipitación de julio a octubre. La duración del experimento fue del mes de agosto a noviembre del 2018. El suelo presenta una textura franco-arcillosoarenoso, $\mathrm{pH}$ de 5.95 y materia orgánica de $4.22 \%$ y conductividad eléctrica de $0.18 \mathrm{dS} \mathrm{m}^{-1}$.

\section{Manejo de la parcela}

El área experimental fue de una superficie de $5000 \mathrm{~m}^{2}$, la cual se preparó con dos pasos de rastra. En el establecimiento en campo, la unidad experimental consistió en una parcela de un metro de ancho por $12.5 \mathrm{~m}$ de largo. Se trazaron tres bloques de manera perpendicular a la pendiente, a fin de contrarrestar el error provocado por la misma. La siembra se realizó de forma manual, con el pasto $M$. maximus $\mathrm{cv}$. aruana, se estableció en tres franjas de cuatro hileras separadas por $20 \mathrm{~cm}$ y un metro entre franja. Se sembró a una densidad de $6 \mathrm{~kg} \mathrm{ha}^{-1}$ de semilla pura viable. La edad de la pradera al iniciar los muestreos fue de un año, el control de maleza se realizó de forma manual. Al inicio del experimento se homogenizó a una altura de planta de $10 \mathrm{~cm}$ del suelo.

\section{Tratamientos}

Los tratamientos consistieron en cuatro diferentes esquemas de fertilización; 1) inorgánica 120-60-00 de NPK, 2) lombricomposta con una dosis de $10 \mathrm{t} \mathrm{ha}^{-1}$, 3) composta con dosis de $10 \mathrm{t} \mathrm{ha}^{-1}$; y 4) composta $\left(10 \mathrm{t} \mathrm{ha}^{-1}\right)$ más una dosis de 2000 litros por hectárea de lixiviado al follaje, dividida en tres aplicaciones, a intervalos de siete días entre cada aplicación. Los insumos para los tratamientos de origen orgánico fueron elaborados en la Unidad Gestora de la Universidad Autónoma Chapingo, de acuerdo a la Norma mexicana NMX-AA-180-SCFI2018 (SE 2018).

\section{Variables evaluadas:}

Materia seca, altura, composición morfológica, relación hoja: tallo

Veinte días posteriores al corte de homogeneización se realizaron muestreos cada catorce días al azar, para estimar la producción de forraje con un cuadro de $0.5 \mathrm{~m} \times 0.5 \mathrm{~m}$, se cortó todo el forraje verde dentro del mismo, a una altura de 10 $\mathrm{cm}$ y se pesó. Del forraje cosechado se tomó una submuestra de $100 \mathrm{~g}$, que se separó en hoja, tallo, espiga y material muerto. El resto del forraje y sus componentes separados se sometió a un secado en una estufa de aire caliente a una temperatura de 55 ${ }^{\circ} \mathrm{C}$ hasta peso constante. Así mismo, en cada corte, se tomaron la altura con una regla a 10 plantas para tener un promedio de cada parcela.

\section{Análisis bromatológico}

En cada corte y tratamiento, se tomaron muestras de materia seca y se determinó por triplicado el contenido de proteína cruda (PC) y cenizas (Ce) con los métodos descritos por la AOAC (2005). La fibra detergente neutro (FDN) y la fibra detergente ácido (FDA) por el método descrito por Van Soest et al. (1993).

\section{Evaluación de la producción de gas in vitro $(\mathrm{mL}$ $\mathbf{h}^{-1}$ )}

Se preparó un medio de cultivo de acuerdo con lo descrito por Herrera-Pérez et al. (2018). Se construyeron biodigestores usando la metodología descrita por Torres-Salado et al. (2019), en los cuales se cambió la fuente de carbono por uno de los tratamientos en estudio. La producción de biogás a las 24,48 y $72 \mathrm{~h}$ se midió mediante el desplazamiento del émbolo de una jeringa de vidrio de $50 \mathrm{~mL}$; BD Yale ${ }^{\circledR}$, Brasil (Hernández-Morales et al. 2018). Los valores de la producción de biogás acumulada se usaron para estimar la cinética de producción de biogás mediante el modelo Gompertz (Lavrencic et al. 1997). Los parámetros $A, b$ y $k$ fueron estimados mediante un análisis de regresión no lineal, utilizando el procedimiento PROC NLMIXED del paquete estadístico SAS (2002). El modelo utilizado fue $\mathrm{Y}=\mathrm{A} \exp [-\mathrm{b}][\exp (-\mathrm{k} t)]$; donde: $\mathrm{Y}=$ volumen de biogás en el tiempo $\mathrm{t}\left(\mathrm{mL} \mathrm{g}^{-1}\right.$ de $\left.\mathrm{MS}\right)$; A: potencial de producción de biogás total cuando t: $\propto\left(\mathrm{mL} \mathrm{g}^{-1}\right.$ de MS); $b$ : tasa constante de producción de biogás del material potencialmente degradable $\left(\mathrm{mL} \mathrm{h}^{-1}\right) ; k$ : tiempo lag (h), factor constante de eficiencia microbiana, definido como el intercepto del eje tiempo de la línea de la tangente en el punto de inflexión; $t$ = 
tiempo de incubación.

A las $72 \mathrm{~h}$ de fermentación se determinaron las características fermentativas in vitro. Para nitrógeno amoniacal $\left(\mathrm{N}^{-\mathrm{NH}_{3}}\right)$ se siguió la metodología de McCullough (1967) modificado por Herrera-Pérez et al. (2018). La degradación de la materia seca (DMS) se calculó con la metodología descrita por HernándezMorales et al. (2018) y Torres-Salado et al. (2019); mientras la degradación de la FDN (DFDN) fue según lo descrito por Hernández-Morales et al. (2018).

\section{Análisis estadístico}

El diseño experimental fue bloques completos al azar, en arreglo de medidas repetidas en el tiempo. Las variables evaluadas se analizaron con los procedimientos Proc Mixed de SAS (SAS 2017), se realizó un análisis de varianza y una comparación de medias Tukey $(p \leq 0.05)$. Las curvas ajustadas de crecimiento con su modelo y coeficientes respectivos para cada tipo de fertilización evaluada, se obtuvieron con el programa Curve Expert Professional 2.0. El análisis químico y el ensayo in vitro se analizaron en un diseño completamente al azar con un arreglo factorial $6 \times 4$, considerando como factores DDC (20, $35,50,65,80,95$ días) y el tipo de fertilización (composta, lombricomposta, químico y composta más lixiviado) con el procedimiento GLM de SAS (SAS 2017). La prueba de comparación de medias fue con la prueba Tukey $(\mathrm{p} \leq 0.05)$.

\section{RESULTADOS}

La máxima acumulación de materia seca en la fertilización inorgánica se presentó a los 80 DDC, con valor de $24013 \mathrm{~kg} \mathrm{ha}^{-1}$, posterior a esa fecha existe una caída, derivado principalmente al acame que sufrió el pasto a consecuencia de las lluvias con fuertes vientos y por la altura de las plantas (Figura 1). La cantidad de hoja con respecto al tallo fue mayor desde los 20 hasta los 65 DDC, encontrándose al día 50 DDC la mejor relación hoja:tallo $\left(6651.7 \mathrm{~kg} \mathrm{ha}^{-1}\right.$ hoja y $4701.6 \mathrm{~kg} \mathrm{ha}^{-1}$ tallo; valor de la relación hoja: tallo) y fue en la fecha 65 DDC cuando se encontró la mayor cantidad de hoja; sin embargo, la cantidad de tallo y material muerto igual se incrementó $(7893 \mathrm{~kg}$ $\mathrm{ha}^{-1}$ de hoja, $6386 \mathrm{~kg} \mathrm{ha}^{-1}$ de tallo y $547 \mathrm{~kg} \mathrm{ha}^{-1}$ de $\mathrm{MM})$. La fertilización con composta más lixiviado presentó la máxima acumulación de materia seca a los 80 DDC, con un rendimiento de $10974 \mathrm{~kg} \mathrm{ha}^{-1}$ y fue a los 65 DDC que se presentó el máximo rendimiento de hojas (4 $825 \mathrm{~kg} \mathrm{ha}^{-1}$ de hoja y $2253 \mathrm{~kg} \mathrm{ha}^{-1}$ de tallo). Pero al día 50 después del corte presentó una mejor relación hoja: tallo (4 $686 \mathrm{~kg} \mathrm{ha}^{-1}$ hoja y 1654 $\mathrm{kg} \mathrm{ha}^{-1}$ de tallo) (Figura 2). La acumulación de materia seca con el tratamiento de lombricomposta se incrementó conforme avanzaron los días al corte, encontrándose el máximo rendimiento a los 95 DDC con $10122 \mathrm{~kg} \mathrm{ha}^{-1}$, destacando que aún en esta fecha, la proporción de hoja encontrada fue alta con relación a los otros tratamientos evaluados con $4841 \mathrm{~kg} \mathrm{ha}^{-1}$ y $4441 \mathrm{~kg} \mathrm{ha}^{-1}$ de tallo (Figura 3); pero la mejor relación hoja: tallo se encontró a los 65 DDC (5 173 $\mathrm{kg} \mathrm{ha}^{-1}$ hoja y $1449 \mathrm{~kg} \mathrm{ha}^{-1}$ tallo). Al igual que con el tratamiento de lombricomposta, el de composta obtuvo la máxima acumulación en el último muestreo a los 95 DDC con un total de $5907 \mathrm{~kg} \mathrm{ha}^{-1}$, destacando que la cantidad de hoja con relación al tallo fue 2.7 mayor (4 $368 \mathrm{~kg} \mathrm{ha}^{-1} \mathrm{~d}$ de hoja por $1177 \mathrm{~kg} \mathrm{ha}^{-1}$ de tallo) por lo que coincidió la máxima acumulación de forraje con la mejor relación hoja: tallo (Figura 4).

El tratamiento con fertilización química mostró diferencia estadística $(p \leq 0.05)$ desde los 50 DDC, ya que mientras los otros tratamientos presentaron alturas de 107, 131 y $121 \mathrm{~cm}$, correspondientes a composta, lombricomposta y composta más lixiviado, respectivamente; el tratamiento químico presentó 175 $\mathrm{cm}$ de altura (Tabla 1). La mayor altura del pasto aruana se presentó en el tratamiento químico a los 80 días después del corte con $189 \mathrm{~cm}$. En la última fecha de corte el tratamiento que menos altura alcanzó fue el de composta más lixiviado con $137 \mathrm{~cm}$; el tratamiento con composta alcanzó los $143 \mathrm{~cm}$ y en la fertilización con lombricomposta se obtuvo una altura de $155 \mathrm{~cm}$.

Las variables químicas evaluadas presentaron interacción entre el tipo de fertilización y los DDC ( $p$ $\leq 0.05$; Tabla 2). El contenido de proteína cruda (PC) en los cuatro tipos de fertilización disminuye conforme aumenta la edad del pasto, a los 20 DDC los valores de PC fueron de 14.5, 14.37, 13.22 y $17.42 \%$, 


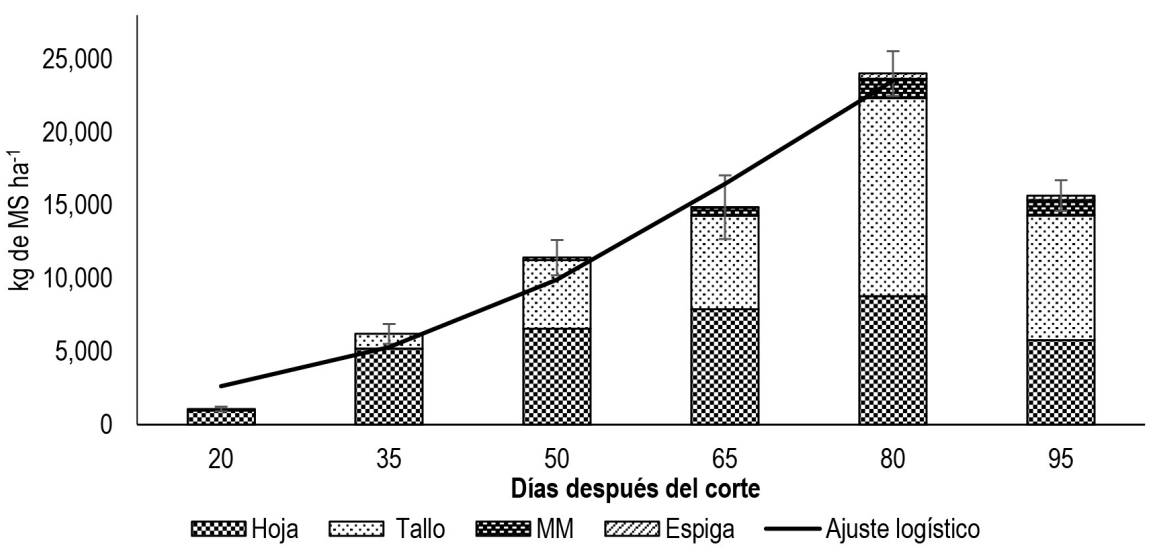

Figura 1. Acumulación de materia seca y composición morfológica del pasto Aruana bajo un sistema de fertilización química.

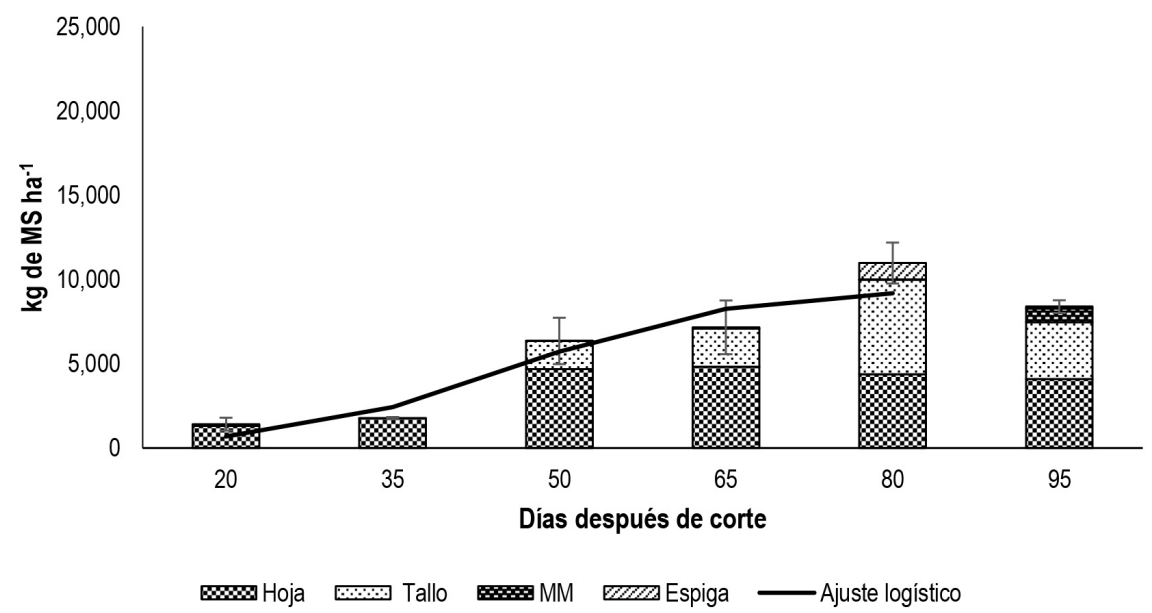

Figura 2. Acumulación de materia seca y composición morfológica del pasto Aruana bajo un sistema de fertilización con composta más lixiviado.

para composta, composta más lixiviado, lombricomposta y tratamiento químico, respectivamente ( $\mathrm{p} \leq$ 0.05) (Tabla 3). En la fecha $35 \mathrm{DDC}$, los tipos de fertilización con composta y lombricomposta redujeron el nivel de proteína a casi la mitad con respecto a la fecha DDC 20 días, mientras que composta más lixiviado y el tratamiento químico disminuyeron el nivel en $25 \%$. En las fechas posteriores no se encontraron diferencias entre tipos de fertilización $(p \leq$ 0.05). Con relación al contenido de fibra detergente neutro (FDN), fibra detergente ácido (FDA) y cenizas se incrementaron a medida que creció el pasto en cada tipo de fertilización ( $p \leq 0.05$, Tabla 3 ).
En el ensayo in vitro, todas las variables presentaron interacción entre el tipo de fertilización y DDC del pasto aruana ( $\leq \leq 0.05$, Tabla 2). A las 48 $\mathrm{h}$, la producción acumulada fue similar entre tipos de fertilización a los 20, 35, 50, 80 y 95 DDC ( $p>0.05)$; sin embargo, a los 65 días, el pasto aruana fertilizado con lombricomposta produjo 2.9 veces mayor biogás que el fertilizado con químicos ( $\leq \leq 0.05)$. En los DDC 20, 35, 50 y 80 no hubo diferencias en la producción de biogás a las $72 \mathrm{~h}$ de fermentación ( $p$ > 0.05); mientras, a los 95 días, el pasto fertilizado con composta más lixiviado produjo 2.4 veces más biogás que el fertilizado con composta ( $p \leq 0.05)$. 


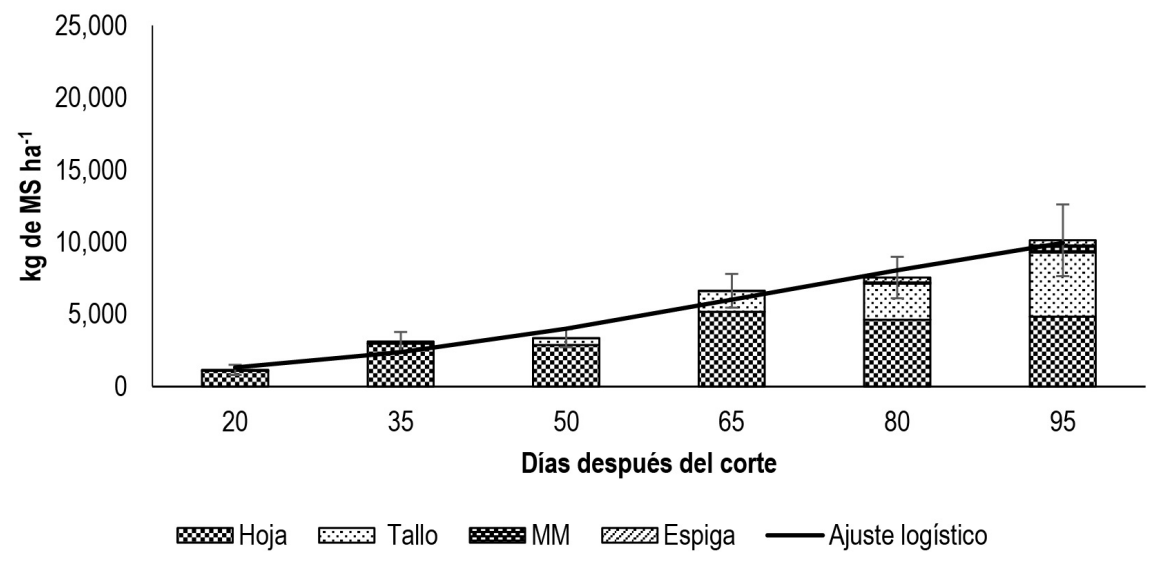

Figura 3. Acumulación de materia seca y composición morfológica del pasto Aruana bajo un sistema de fertilización con lombricomposta.

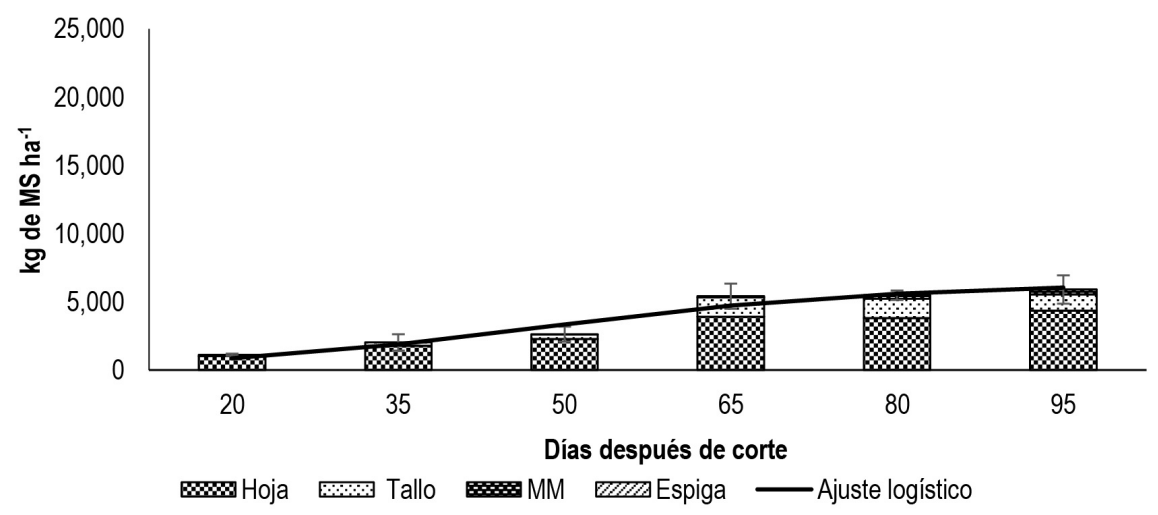

Figura 4. Acumulación de materia seca y composición morfológica del pasto Aruana bajo un sistema de fertilización con composta.

Tabla 1. Alturas en centímetros del pasto Aruana en diferentes etapas de corte bajo diferentes esquemas de fertilización.

\begin{tabular}{lllllll}
\hline \multirow{2}{*}{ Fertilización } & \multicolumn{6}{c}{ Días después del corte } \\
\cline { 2 - 7 } & 20 & 35 & 50 & 65 & 80 & 95 \\
\hline Composta & $44^{a}$ & $84^{a}$ & $107^{a}$ & $138^{a}$ & $143^{a}$ & $143^{a}$ \\
Lombricomposta & $42^{a}$ & $90^{a}$ & $131^{a b}$ & $147^{a}$ & $155^{a}$ & $155^{a}$ \\
Composta + lixiviado & $39^{a}$ & $88^{a}$ & $121^{a b}$ & $138^{a}$ & $143^{a}$ & $137^{a}$ \\
Químico & $43^{a}$ & $115^{a}$ & $175^{b}$ & $185^{b}$ & $189^{b}$ & $186^{b}$ \\
\hline
\end{tabular}

$\overline{a, b}$ Literales diferentes dentro de cada columna, indican diferencia significativa $(P \leq 0.05)$.

\section{DISCUSIÓN}

El máximo rendimiento de materia seca encontrado en el tratamiento químico a los 80 DDC (24 $013 \mathrm{~kg} \mathrm{ha}^{-1}$ ) fue mayor a lo reportado en literatura con promedios de 15000 a $20000 \mathrm{~kg} \mathrm{ha}^{-1}$ año $^{-1}$ (Castrejón et al. 2017). Así mismo, Fernández et al. (2015), reportan un rendimiento de materia seca de $13800 \mathrm{~kg} \mathrm{ha}^{-1}$, en lluvias con $1200 \mathrm{~mm}$ de precipitación anual y una fertilización inorgánica de 10-25-27 de NPK. Mientras que Santana et al. (2020) encontraron un rendimiento de tan solo 2 $775 \mathrm{~kg} \mathrm{MS} \mathrm{ha}^{-1}$, en la época seca con fertilización química. El rendimiento de materia seca obtenido 
Tabla 2. Valor de $\mathrm{p}$ de las variables químicas y producción de gas in vitro de pasto aruana a diferente edad de rebrote con diferentes fuentes de fertilización.

\begin{tabular}{|c|c|c|c|}
\hline Variable & Fertilización & Días después de corte & Fertilización`Días después de corte \\
\hline Materia orgánica & $<0.0001$ & $<0.0001$ & 0.0003 \\
\hline Proteína cruda & 0.0001 & $<0.0001$ & $<0.0001$ \\
\hline Fibra detergente neutro & $<0.0001$ & $<0.0001$ & $<0.0001$ \\
\hline Fibra detergente ácido & 0.0941 & $<0.0001$ & 0.0008 \\
\hline Cenizas & $<0.0001$ & $<0.0001$ & 0.0003 \\
\hline Biogás a las 24 h & $<0.0001$ & 0.0145 & 0.0131 \\
\hline Biogás a las $48 \mathrm{~h}$ & $<0.0001$ & 0.0031 & 0.0279 \\
\hline Biogás a las $72 \mathrm{~h}$ & $<0.0001$ & 0.0002 & 0.055 \\
\hline Producción de biogás total (A) & $<0.0001$ & $<0.0001$ & $<0.0001$ \\
\hline Tasa de producción de biogás $(b)$ & $<0.0001$ & $<0.0001$ & $<0.0001$ \\
\hline Tiempo lag $(K)$ & 0.015 & 0.6187 & $<0.0001$ \\
\hline Degradación de materia seca & $<0.0001$ & $<0.0001$ & $<0.0001$ \\
\hline Degradación de fibra detergente neutro & $<0.0001$ & $<0.0001$ & $<0.0001$ \\
\hline Nitrógeno amoniacal & 0.0012 & $<0.0001$ & 0.0103 \\
\hline
\end{tabular}

Tabla 3. Materia orgánica, proteína cruda, fibras detergentes y cenizas de pasto aruana a diferente edad de rebrote con diferentes fuentes de fertilización.

\begin{tabular}{|c|c|c|c|c|c|c|}
\hline Fertilización & DDC & $\mathrm{MO}(\%)$ & PC (\%) & FDN (\%) & FDA (\%) & $\mathrm{Ce}(\%)$ \\
\hline \multirow{5}{*}{ Composta } & 20 & $88.4^{e}$ & $14.5^{b c}$ & $65.7^{k l}$ & $37.5^{\text {bcdef }}$ & $11.5^{b}$ \\
\hline & 35 & $89.5^{d e}$ & $7.2^{f g h}$ & $69.1^{j k}$ & $38.1^{b c d e f}$ & $10.5^{b c}$ \\
\hline & 50 & $89.7^{d e}$ & $8.5^{e f}$ & $69.8^{i j}$ & $38.9^{b c d e f}$ & $10.2^{b c}$ \\
\hline & 65 & $90.0^{d e}$ & $7.4^{f g h}$ & $73.1^{\text {efghi }}$ & $40.7^{b c d e f}$ & $9.9^{b c}$ \\
\hline & 95 & $89.8^{d e}$ & $8.4^{e f g}$ & $73.4^{e f g h}$ & $41.3^{\text {bcdef }}$ & $10.1^{b c}$ \\
\hline \multirow{6}{*}{$\begin{array}{l}\text { Composta + } \\
\text { Lixiviado }\end{array}$} & 20 & $88.5^{e}$ & $14.3^{b c d}$ & $70.5^{g h i j}$ & $38.4^{b c d e f}$ & $11.5^{b}$ \\
\hline & 35 & $89.3^{d e}$ & $11.1^{d e}$ & $70.0^{h i j}$ & $37.1^{\text {cdef }}$ & $10.7^{b c}$ \\
\hline & 50 & $89.5^{d e}$ & $5.6^{f g h}$ & $74.6^{\text {def }}$ & $42.1^{b c d e f}$ & $10.4^{b c}$ \\
\hline & 65 & $90.1^{d e}$ & $8.5^{e f}$ & $74.1^{d e f g}$ & $41.4^{b c d e f}$ & $9.8^{b c}$ \\
\hline & 80 & $90.8^{d e}$ & $7.3^{f g h}$ & $73.6^{e f g}$ & $41.3^{b c d e f}$ & $9.1^{b c}$ \\
\hline & 95 & $91.1^{d e}$ & $7.4^{f g h}$ & $77.4^{b c d}$ & $46.6^{b c}$ & $8.8^{b c}$ \\
\hline \multirow{6}{*}{ Lombricomposta } & 20 & $89.5^{d e}$ & $13.2^{c d}$ & $65.3^{l}$ & $34.7^{e f}$ & $10.4^{b c}$ \\
\hline & 35 & $92.6^{c d e}$ & $7.1^{f g h}$ & $70.6^{g h i j}$ & $39.4^{b c d e f}$ & $7.3^{b c d}$ \\
\hline & 50 & $98.6^{b}$ & $6.3^{f g h}$ & $79.1^{b c}$ & $43.5^{b c d e}$ & $1.3^{e}$ \\
\hline & 65 & $97.3^{b c}$ & $7.5^{f g h}$ & $79.9^{b}$ & $45.9^{b c}$ & $2.6^{d e}$ \\
\hline & 80 & $93.8^{b c d}$ & $4.9^{h}$ & $78.9^{b c}$ & $45.4^{b c}$ & $6.1^{c d e}$ \\
\hline & 95 & $89.6^{d e}$ & $6.9^{f g h}$ & $74.4^{\text {def }}$ & $34.8^{\text {def }}$ & $10.3^{b c}$ \\
\hline \multirow{6}{*}{ Químico } & 20 & $88.0^{e}$ & $17.4^{b}$ & $61.5^{\mathrm{m}}$ & $33.0^{f}$ & $11.9^{b}$ \\
\hline & 35 & $89.8^{d e}$ & $12.7^{c d}$ & $71.2^{f g h i j}$ & $40.0^{\text {bcdef }}$ & $10.1^{b c}$ \\
\hline & 50 & $92.0^{d e}$ & $8.1 \mathrm{e}^{f g h}$ & $75.6^{c d e}$ & $43.8^{b c d e}$ & $7.9^{b c}$ \\
\hline & 65 & $92.2^{\text {de }}$ & $5.6^{f g h}$ & $75.6^{\text {cde }}$ & $44.2^{b c d}$ & $7.7^{b c}$ \\
\hline & 80 & $92.2^{\text {cde }}$ & $5.2^{g h}$ & $75.3^{\text {de }}$ & $43.8^{b c d e}$ & $7.7^{b c d}$ \\
\hline & 95 & $90.4^{d e}$ & $5.0^{h}$ & $74.1^{\operatorname{defg}}$ & $46.8^{b}$ & $9.5^{b c}$ \\
\hline Error estándar de la media & 0.35 & 0.42 & 0.55 & 0.55 & 0.35 & \\
\hline
\end{tabular}

con la aplicación de composta más lixiviado en este experimento es similar al obtenido por Álvarez et al. (2016), quienes fertilizaron al pasto M. maximus con diferentes dosis de lixiviado de composta de cerdo, en el que su rendimiento fue de $4950 \mathrm{~kg} \mathrm{ha}^{-1}$, a los 50 días después de corte de homogenización, y en esta evaluación a la misma fecha de muestreo se obtuvieron $6349 \mathrm{~kg} \mathrm{ha}^{-1}$. Los altos rendimien- tos encontrados están posiblemente relacionados a que este pasto mejorado requiere suelos fértiles para su buen desarrollo (Castrejón et al. 2017, Santana et al. 2020), por lo que tuvo una buena respuesta a los diferentes tipos de fertilización. Al respecto, Álvarez et al. (2016) concluyeron que el rendimiento, la calidad y los ingresos por producción se incrementaron con el nivel de fertilización utilizado en el pasto 
M. maximus cuando este se evaluó con fertilización química y orgánica ya que la especie se caracteriza por su gran potencial de producción de biomasa y calidad cuando son agronómicamente bien manejados. Es importante mencionar que la fertilización nitrogenada conlleva modificaciones en la calidad nutricional de las pasturas, las cuales no son visibles para los productores, pero generan muchos efectos negativos en los niveles productivos, económico y ambiental, por lo que las fertilizaciones orgánicas representan una opción agroecológica (Álvarez et al. 2016). Los resultados del análisis bromatológico en la presente investigación y los reportados en la literatura son consecuencia del manejo agronómico en que se producen (Valenciaga y Chongo 2004, Barahona et al. 2003) y la edad de corte, porque se incrementa la pared celular y el contenido de lignina (Gándara et al. 2017). La cantidad de proteína cruda es similar a la reportada por otros autores como Verdecia et al. 2008, quienes analizaron al pasto M. maximus cv. Tanzania, en las mismas fechas de corte que en este experimento, encontrando porcentajes de proteína que disminuían de acuerdo avanzaba la fecha de corte de la pradera, de $11.25 \%$ a los 20 DDC hasta $5.56 \%$ a los 95 DDC. Mientras que Castrejón et al. (2017) encontraron niveles más bajos de proteína a los $21,42,63$ y 74 DDC con 7.9, 7.7, 9.8 y $6.3 \%$, respectivamente, posiblemente relacionado a que no eran pastos fertilizados y a la deficiente adaptación de la variedad a las condiciones del suelo y el ambiente (Enríquez et al. 2011). La FDN y FDA se incrementaron conforme avanzó la edad del pasto. En tanto que Castrejón et al. (2017) al evaluar el pasto aruana a los 63 DDC encontraron $75.21 \%$ de FDN en el pasto $\sin$ fertilizar siendo muy similar a lo reportado en esta investigación con $74.45 \%$ como promedio de los cuatro tratamientos evaluados; sin embargo, la FDA fue de $57.27 \%$ por 40.7 que promediaron los tratamientos evaluados por lo que hubo una diferencia en cuanto a la lignina y celulosa, ya que estas son menores independientemente del tipo de fertilización.

La producción de biogás muestra la disponibilidad de los carbohidratos para la fermentación por los microorganismos ruminales (Texta-Nogueda et al. 2019). Así, a las 24 h se mostró variación en la producción de biogás; pero al fijar por día de rebrote, tenemos que a los 20, 35, 65 y 80 DDC no hubo diferencias entre tipo de fertilización $(p>$ $0.05)$; en contraste a los 50 y $95 \mathrm{DDC}$, el pasto aruana fertilizado con lombricomposta o composta más lixiviado produjeron 2.8 y 2.0 veces mayor biogás que el fertilizado con químicos o composta $(p \leq 0.05)$. Esto indica una mayor disponibilidad de carbohidratos no estructurales en el pasto aruana fertilizado con lombricomposta o composta más lixiviado, ya que en las primeras $24 \mathrm{~h}$ se fermentan este tipo de carbohidratos (Torres-Salado et al. 2019). Además, al día 65 de rebrote, el pasto fertilizado con lombricomposta produjo 2.2 veces más biogás que el fertilizado con químicos $(p \leq 0.05)$. Esto se asume porque a partir de las $48 \mathrm{~h}$ se fermentan los carbohidratos estructurales (Torres-Salado et al. 2019), por lo que el uso de fertilizante modificó la composición química y disponibilidad de los carbohidratos del pasto aruana a los 65 y 95 DDC (Tabla 4).

En el caso de la producción de biogás evaluada a diferentes tiempos, a las $72 \mathrm{~h}$ fue mayor comparada con la reportada por otros autores como Almaraz-Buendía et al. (2019), quienes evaluaron la producción acumulada de biogás en los pastos bermuda (Cynodon dactylon), mulato (Brachiaria híbrida), insurgente (Brachiaria brizantha), estrella (Cynodon nlemfuensis), bramilla (Elytrichia repens), llanero (Andropogon gayanus), mombaza (M. maximus), pará (Brachiaria mutica) y pangola (Digitaria decumbens) con 60 días de rebrote sin fertilización. Los parámetros de la cinética de producción de biogás permiten establecer la fase del crecimiento de los microorganismos ruminales durante la fermentación del pasto aruana. El parámetro A es un indicativo de cuando los microrganismos alcanzan su fase estacionaria (Rojas-García et al. 2020), por lo que este no mostró diferencias entre tipos de fertilización a los 50 y 80 DDC ( $p>$ 0.05). A los 20 días, la fertilización con composta mostró $30.20 \%$ mayor A que composta más lixiviado; a los 35 días, la lombricomposta produjo mayor $\mathrm{A}$; a los 65 días, composta más lixiviado y el tratamiento químico produjeron $46.27 \%$ más A que los otros tipos de fertilización; y a los 95 días, la composta más 
Tabla 4. Producción de biogás, cinética de fermentación y características fermentativas in vitro de pasto aruana a diferentes edades de rebrote fertilizado con diferentes productos.

\begin{tabular}{|c|c|c|c|c|c|c|c|c|c|c|}
\hline Fertilización & DDC & $\begin{array}{r}\text { Biogás24 } \\
\left(\mathrm{mL} \mathrm{g}^{-1} \mathrm{MS}\right)\end{array}$ & $\begin{array}{r}\text { Biogás48 } \\
\left(\mathrm{mL} \mathrm{g}^{-1} \mathrm{MS}\right)\end{array}$ & $\begin{array}{r}\text { Biogás72 } \\
\left(\mathrm{mL} \mathrm{g}^{-1} \mathrm{MS}\right)\end{array}$ & $\begin{array}{r}\mathrm{A} \\
\left(\mathrm{mL} \mathrm{g}{ }^{-1} \mathrm{MS}\right)\end{array}$ & $\begin{array}{r}k \\
\text { (h) }\end{array}$ & $\begin{array}{r}b \\
\left(m L h^{-1}\right)\end{array}$ & $\begin{array}{r}\text { DMS } \\
(\%)\end{array}$ & $\begin{array}{r}\text { DFDN } \\
(\%)\end{array}$ & $\begin{array}{r}\mathrm{N}-\mathrm{NH}_{3} \\
\left(\mathrm{mg} \mathrm{dL}^{-1}\right)\end{array}$ \\
\hline \multirow{5}{*}{ Composta } & 20 & $89.5^{a b c}$ & $146.9^{a b c d}$ & $160.7^{a b c d}$ & $218.6^{b c}$ & $3.10^{\text {def }}$ & $0.100^{a b}$ & $78.3^{b c d}$ & $78.1^{b c}$ & $0.87^{b}$ \\
\hline & 35 & $36.0^{a b}$ & $61.0^{a b c}$ & $90.1^{a b c}$ & $145.1^{e f g}$ & $2.36^{f g}$ & $0.035^{a b}$ & $30.9^{l}$ & $18.1^{m}$ & $0.73^{b}$ \\
\hline & 50 & $35.9^{a b}$ & $96.9^{a b c d}$ & $151.0^{a b c d}$ & $175.9^{\text {cdef }}$ & $4.44^{c d}$ & $0.047^{a b}$ & $60.2^{i}$ & $62.0^{g h i}$ & $0.33^{b}$ \\
\hline & 65 & $36.0^{a b}$ & $90.6^{a b c d}$ & $125.6^{a b c d}$ & $144.9^{e f g}$ & $3.82^{\text {cde }}$ & $0.100^{a b}$ & $66.3^{g h i}$ & $66.8^{d e f g h i}$ & $0.31^{b}$ \\
\hline & 95 & $34.1^{a b}$ & $42.1^{a}$ & $68.5^{a}$ & $61.0^{h}$ & $1.47^{g}$ & $0.036^{a b}$ & $39.0^{k l}$ & $24.2^{l m}$ & $0.73^{b}$ \\
\hline \multirow{6}{*}{ Composta +Lixiviado } & 20 & $55.1^{a b c}$ & $103.8^{a b c d}$ & $149.7^{a b c d}$ & $167.9^{\text {def }}$ & $7.34^{b}$ & $0.100^{a b}$ & $82.6^{b}$ & $82.6^{b}$ & $1.04^{a b}$ \\
\hline & 35 & $57.5^{a b c}$ & $121.6^{a b c d}$ & $162.7^{a b c d}$ & $171.2^{\text {cdef }}$ & $5.25^{c}$ & $0.100^{a b}$ & $75.7^{b c d e f}$ & $77.5^{b c d}$ & $0.50^{b}$ \\
\hline & 50 & $121.2^{c}$ & $181.7^{d}$ & $212.7^{d}$ & $211.6^{b c d}$ & $2.89^{e f g}$ & $0.100^{a b}$ & $71.3^{\text {cdefgh }}$ & $69.2^{\text {cdefghi }}$ & $0.65^{b}$ \\
\hline & 65 & $57.3^{a b c}$ & $117.0^{a b c d}$ & $149.5^{a b c d}$ & $174.7^{c d e f}$ & $3.27^{d e f}$ & $0.044^{a b}$ & $68.6^{e f g h i}$ & 67.1 defghi & $0.39^{b}$ \\
\hline & 80 & $82.7^{a b c}$ & $124.9^{a b c d}$ & $160.1^{a b c d}$ & $171.1^{\text {cdef }}$ & $2.57^{e f g}$ & $0.100^{a b}$ & $69.4^{d e f g h i}$ & $65.6^{f g h i}$ & $0.47^{b}$ \\
\hline & 95 & $77.3^{b c d}$ & $137.1^{a b c d}$ & $166.4^{b c d}$ & $176.0^{\text {cdef }}$ & $2.83^{e f g}$ & $0.200^{b}$ & $62.5^{h i}$ & $60.0^{h i}$ & $0.28^{b}$ \\
\hline \multirow{6}{*}{ Lombricomposta } & 20 & $97.2^{a b c}$ & $158.8^{b c d}$ & $184.0^{c d}$ & $191.5^{\text {cde }}$ & $3.17^{\text {def }}$ & $0.100^{a b}$ & $73.0^{\text {cdefg }}$ & $72.1^{b c d e f g}$ & $0.46^{b}$ \\
\hline & 35 & $70.4^{a b c}$ & $129.1^{a b c d}$ & $177.1^{b c d}$ & $253.0^{b}$ & $3.04^{d e f}$ & $0.041^{a b}$ & $75.1^{b c d e f g}$ & 74.1 bcdef & $0.42^{b}$ \\
\hline & 50 & $103.8^{b c}$ & $142.7^{a b c d}$ & $190.2^{d}$ & $210.0^{b c d}$ & $2.24^{f g}$ & $0.100^{a b}$ & $74.1^{b c d e f g}$ & $76.1^{b c d e f}$ & $0.30^{b}$ \\
\hline & 65 & $87.4^{a b c}$ & $166.9^{c d}$ & $194.2^{d}$ & $206.6^{b c d}$ & $3.09^{d e f}$ & $0.100^{a b}$ & $67.9^{f g h i}$ & $66.3^{e f g h i}$ & $0.39^{b}$ \\
\hline & 80 & $61.9^{a b c}$ & $119.3^{a b c d}$ & $163.0^{a b c d}$ & $201.4^{c d}$ & $2.76^{e f g}$ & $0.042^{a b}$ & $49.0^{j}$ & $46.3^{j}$ & $0.44^{b}$ \\
\hline & 95 & $60.4^{a b c}$ & $106.1^{a b c d}$ & $137.9^{a b c d}$ & $167.7^{\text {def }}$ & $2.93^{e f g}$ & $0.049^{a b}$ & $70.5^{\text {cdefgh }}$ & $70.4^{\text {cdefgh }}$ & $0.57^{b}$ \\
\hline \multirow{6}{*}{ Químico } & 20 & $64.2^{a b c}$ & $139.3^{a b c d}$ & $158.5^{a b c d}$ & $176.9^{\text {cdef }}$ & $3.06^{d e f}$ & $0.100^{a b}$ & $78.9^{b c}$ & $82.8^{b}$ & $1.80^{a}$ \\
\hline & 35 & $62.6^{a b c}$ & $121.7^{a b c d}$ & $148.3^{a b c d}$ & $174.0^{\text {cdef }}$ & $3.33^{d e f}$ & $0.100^{a b}$ & $77.4^{\text {bcde }}$ & $77.3^{\text {bcde }}$ & $0.66^{b}$ \\
\hline & 50 & $43.2^{a b}$ & $98.9^{a b c d}$ & $133.9^{a b c d}$ & $218.6^{b c}$ & $2.54^{e f g}$ & $0.027^{a b}$ & $63.8^{h i}$ & $58.9^{i}$ & $0.39^{b}$ \\
\hline & 65 & $28.3^{a}$ & $56.5^{a b}$ & $88.9^{a b c}$ & $137.6^{\mathrm{fg}}$ & $2.45^{e f g}$ & $0.014^{a b}$ & $44.1^{j k}$ & $40.3^{j k}$ & $0.47^{b}$ \\
\hline & 80 & $31.9^{a}$ & $46.3^{a}$ & $85.2^{a b}$ & $177.3^{\text {cdef }}$ & $2.94^{e f}$ & $0.011^{a b}$ & $38.0^{\mathrm{kl}}$ & $42.0^{j}$ & $0.72^{b}$ \\
\hline & 95 & $33.1^{a}$ & $54.4^{a b}$ & $84.8^{a b}$ & $100.4^{g h}$ & $2.24 f g$ & $0.017^{a}$ & $46.5^{j k}$ & $30.4^{k l}$ & $0.85^{b}$ \\
\hline EEM & 3.61 & 5.48 & 5.14 & 4.50 & 0.13 & 0.006 & 1.79 & 2.25 & 0.05 & \\
\hline
\end{tabular}

lixiviado y lombricomposta produjeron $112.87 \%$ más A que los otros tipos de fertilización $(p \leq 0.05)$. El parámetro $k$ representa el tiempo que requieren los microorganismos para adherirse al sustrato e iniciar el catabolismo (Rojas-García et al. 2020); de modo que, a los 20 y 35 días, el pasto aruana fertilizado con composta más lixiviado mostraron el mayor $k$, mientras a los 50 días, cuando se usó composta como fertilizante presentó el mayor $\mathrm{k}(\mathrm{p} \leq 0.05)$; sin embargo, a los 65,80 y 95 DDC, el tipo de fertilización no infirió en $k(p>0.05)$. El parámetro $b$ representaría la velocidad de fermentación del sustrato que puede compararse con la fase de crecimiento logarítmico de los microorganismos ruminales (RojasGarcía et al. 2020), donde sólo se presentó diferencia a los 95 DDC entre la fertilización con composta más lixiviado y químicos ( $\leq \leq 0.05)$, ya que el resto de los días de rebrote con cualquier tipo de fertilización no mostraron diferencias $(p>0.05)$. Almaraz-Buendía et al. (2019) publicaron una cinética de fermentación de 9 forrajes con 60 DDC usados comúnmente en el trópico de México, donde sus valores fueron menores para $A$ y mayores para $b$ y $k$ en cualquiera de las edades de rebrote del presente estudio.
La mayor degradación de la materia seca (DMS) se presentó a los 20 DDC, y esta disminuyó conforme aumentó la edad de rebrote; sin embargo, por edad de rebrote, a los 20 días el pasto aruana fertilizado con lombricomposta mostró la menor DMS, además a los 35,50 y 95 DDC, la fertilización con composta, así como a los 50, 80 y 95 días, la fertilización con químicos mostraron la menor DMS ( $p$ $\leq 0.05$ ). La mayor degradación de la fibra detergente neutro (DFDN) del pasto aruana fue a los 20 DDC con cualquier fertilización, fertilización con composta + lixiviado, lombricomposta y químicos a los 35 DDC y lombricomposta a los $50 \mathrm{~d}$, sin diferencias entre ellos $(p>0.05$, Tabla 4). Estos valores son indicativos del comportamiento del pasto cuando se ofrezca al animal, ya que valores menores de $40 \%$ en la DFDN, indican disminución en el consumo por parte del animal (Hoffman, 2007). Valores inferiores de DMS y DFDN fueron reportados en 9 pastos tropicales con 60 DDC (Almaraz-Buendía et al. 2019). El contenido de nitrógeno amoniacal indica la degradabilidad de la fracción nitrogenada (Khejornsart et al. 2011). El mayor contenido de nitrógeno amoniacal en el medio de cultivo fue a los $20 \mathrm{~d}$ con la fertilización de quími- 
cos y composta + lixiviado ( $p \leq 0.05$; Tabla 4 ), lo que indica mayor degradación de la proteína contenida en el pasto aruana.

\section{CONCLUSIONES}

La fecha óptima de corte para el pasto aruana se encuentra entre los 50 y 65 DDC, ya que en este periodo presenta un rendimiento de materia seca con elevado porcentaje de proteína y alta proporción de hojas. El pasto aruana fertilizado con composta más lixiviado y lombricomposta mostraron mayor producción de biogás, a los 50 y 95 DDC por lo que indica una mayor disponibilidad de carbohidratos para la fermentación por los microorganismos ruminales. La lombricomposta y composta más lixiviado pueden ser considerados como opciones de fertilizantes accesibles para los productores en el pasto aruana.

\section{AGRADECIMIENTOS}

Al Centro de Investigación en Biología, Educación Ambiental y Agricultura Orgánica (CIBEAO) perteneciente a la Dirección General de Investigación y Posgrado por el financiamiento del proyecto convencional 20089-C-83.

\section{LITERATURA CITADA}

AOAC (2005) Official Methods of Analysis (18th Ed). AOAC International. Washinton D.C. USA. 96p.

Almaraz-Buendía I, García AM, Sánchez-Santillán P, Torres-Salado N, Herrera-Pérez J, Bottini-Luzardo MB, Rojas-García AR (2019) Análisis bromatológico y producción de gas in vitro de forrajes utilizados en el trópico seco mexicano. Archivos de Zootecnia 68: 260-266.

Álvarez PGR, Vargas BJC, Franco CFJ, Álvarez PPE, Samaniego AMC, Moreno MPA, Chacón ME, García MAR, Arana MRSS, Ramíres RJL (2016) Rendimiento y calidad del pasto Megathyrsus maximus fertilizado con residuos líquidos de cerdo. Revista Electrónica de Veterinaria 17: 1-9.

Améndola MR, Castillo GE, Martínez PA (2005) Perfiles por País del Recurso Pastura/ Forraje. México. FAO $57 p$.

Barahona R, Lascano CE, Narvaez N, Owen E, Morris P, Theodorou MK (2003) In vitro degradability of mature and immature leaves of tropical forage legumes differing in condensed tannin and non-starch polysaccharide content and composition. Journal of the Science of Food and Agriculture 83: 1256-1266.

Castrejón PFA, Corona GL, Rosiles ML, Martínez PP, Lorenzana MAV, Arzate VLG, Olivos AP, Guzmán SS, García PA, Avilés NJN, et al. (2017) Características nutrimentales de gramíneas, leguminosas y algunas arbóreas del Trópico Mexicano: Fracciones de proteína (A, B1, B2, B3 y C), carbohidratos y digestibilidad in vitro. Universidad Nacional Autónoma de México 171p.

Enríquez QJF, Meléndez NF, Bolaños AED, Esqueda EVA (2011) Producción y manejo de forrajes tropicales. Instituto Nacional de Investigaciones Agrícolas y Pecuarias, Centro de Investigación Regional Golfo Centro, Campo Experimental La Posta, Primera Edición. Medellín de Bravo, Veracruz, México. 405p.

Fernández D, López M, Pérez Y, Arzola J, de-La-Fe G (2015) Evaluación del comportamiento en corte de cultivares de los géneros Megathyrsus y Brachiarias introducidas en suelo Ferralítico rojo. Ecosistema Ganadero 2: 27-34.

Flores AJJ, Vázquez RR, Solano VJJ, Aguirre FV, Flores PFI, Fernando IBG, Oliver-Guadarrama R, GranjenoColín AE, Orihuela-Trujillo A (2012) Efecto de fertilizante orgánico, inorgánico y su combinación en la producción de alfalfa y propiedades químicas del suelo. Terra Latinoamericana 30: 213-220. 
Gándara L, Borrajo Cl, Fernández JA, Pereira MM (2017) Efecto de la fertilización nitrogenada y la edad del rebrote sobre el valor nutritivo de Brachiaria brizantha cv. Marandu. Revista FCA Uncuyo 49: 69-77.

Hernández-Morales J, Sánchez-Santillán P, Torres-Salado N, Herrera-Pérez J, Rojas-García AR, Reyes-Vázquez I, Mendoza-Núñez MA (2018) Composición química y degradaciones in vitro de vainas y hojas de leguminosas arbóreas del trópico seco de México. Revista Mexicana de Ciencias Pecuarias 9: 105-120.

Herrera-Pérez JL, Vélez-Regino G, Sánchez-Santillán P, Torres-Salado N, Rojas-García AR, Maldonado-Peralta $\mathrm{M}$ (2018) In vitro fermentation of fibrous substrates by wáter buffalo ruminal cellulolytic bacteria consortia. Revista MVZ Cordoba 23: 6860-6870.

Hoffman PC, Lundberg KM, Bauman LM, Shaver RD, Contreras GFE (2007) El efecto de la madurez en la digestibilidad del FDN (fibra detergente neutro). Focus Forage 5: 1-2.

Jiménez OMM, Granados L, Oliva J, Quiroz J, Barrón M (2010) Calidad nutritiva de Brachiaria humidicola con fertilización orgánica e inorgánica en suelos ácidos. Archivos de Zootecnia 59: 561-570.

Khejornsart P, Wanapat M, Rowlinson P (2011) Diversity of anaerobic fungi and rumen fermentation characteristic in swamp buffalo and beef cattle fed on different diets. Livestock Science 139: 230-236.

Lavrenčič A, Stefanon B, Susmel P (1997) An evaluation of the Gompertz model in degradability studies of forage chemical components. Animal Science 64: 423-431.

McCullough H (1967) The determination of ammonia in whole blood by a direct colorimetric method. Clinica Chimica Acta 17: 297-304.

Muñoz-González JC, Huerta-Bravo M, Lara-Bueno A, Rangel-Santos R, de la Rosa-Arana JL (2016) Producción y calidad nutrimental de forrajes en condiciones del Trópico Húmedo de México. Revista Mexicana de Ciencias Agrícolas 16: 3315-3327.

SE (2018) Norma Mexicana NMX-AA-180-SCFI-2018 Que establece los métodos y procedimientos para el tratamiento aerobio de la fracción orgánica de los residuos sólidos urbanos y de manejo especial, así como la información comercial y de sus parámetros de calidad de los productos finales. https://biblioteca.semarnat. gob.mx/janium/Documentos/Ciga/agenda/PPD1/NMX-AA-180-SCFI-2018.pdf. Fecha de consulta: 20 de enero de 2021.

Quero CAR (2013) Gramíneas introducidas: Importancia e impacto en ecosistemas ganaderos. Primera edición. Editorial del Colegio de Postgraduados. México. 167p.

Quero-Carrillo AR, Enríquez-Quiroz JF, Morales-Nieto CR, Miranda-Jiménez L (2010) Apomixis y su importancia en la selección y mejoramiento de gramíneas forrajeras tropicales. Revisión. Revista Mexicana de Ciencias Pecuarias 1: 25-42.

Ramírez-Meléndez JE, Hernández-Guzmán FJ, López-Castañeda C, Miranda-Jiménez L, Carrillo-Llanos MJ, Quero-Carrillo AR (2020) Crecimiento de plántulas de nueve genotipos de Bouteloua curtipendula con dos tamaños de cariópside. Revista Fitotecnia Mexicana 43: 171-180.

Rojas-García AR, Orocio-Martínez RK, Sánchez-Santillan SP, Ayala-Monter MA, Maldonado-Peralta MA, Valenzuela-Lagarda JL (2020) Características bromatológicas y fermentativas in vitro de complementos con Enterolobium cyclocarpum (Jacq.) Griseb y cáscara de Cucurbita argyrosperma Huber. Agroproductividad 13: 67-74.

Sánchez DE, Arreaza, LC, Abadía B (2005) Estudio de la cinética de degradación in vitro de cuatro forrajeras tropicales y una leguminosa de clima templado. Revista Corpoica 6: 58-68. 
Santana FL, dos Santos DG, Geraldo CM, Emerenciano MJV, Medeiros AIM, Santos GJL, Chaves GAL (2020) Estructura del pasto, y rendimiento de ovejas suplementadas con diferentes pastos tropicales en la estación seca. Revista Mexicana de Ciencias Pecuarias 11: 89-101.

SAS (2017) SAS/STAT User's Guide. Release 6. SAS Institute. Cary, NC, USA. 1848p.

Texta-Nogueda J, Sánchez-Santillan P, Hernández-Sánchez D, Torres-Salado N, Crosby-Galván MM, RojasGarcía AR, Herrera-Pérez J, Maldonado-Peralta M (2019) Use of disaccharides and activated carbon to preserve cellulolytic ruminal bacterial consortiums lyophilized. Revista MVZ Cordoba 24: 7305-7313.

Torres-Salado N, Sánchez-Santillán P, Rojas-García AR, Almaraz-Buendia I, Herrera-Pérez J, Reyes Vázquez I, Mayren-Mendoza J (2019) In vitro gas production and fermentative characteristics of ruminal cellulolytic bacterial consortia of water buffalo (Bubalus bubalis) and Suiz-bu cow. Agrociencia 53: 145-159.

Valenciaga D, Chongo B (2004) La pared celular. Influencia de su naturaleza en la degradación microbiana ruminal de los forrajes. Revista Cubana de Ciencia Agrícola 38: 343-350.

Van Soest PJ, Alien MS (1993) Limitations of prediction systems for digestibility and ration balancing. Silage Production from Seed to Animal. NRAES. Ithaca, N.Y. USA. pp: 196-210.

Verdecia DM, Ramírez LJ, Leonard I, Pascual Y, López Y (2008) Rendimiento y componentes del valor nutritivo del Panicum cv. Tanzania. Revista electrónica de Veterinaria 9: 1-9. 